\title{
Postpartum Depression and Cognitive Behavioral Therapy from Face to Face Group Sessions to Online Group Sessions
}

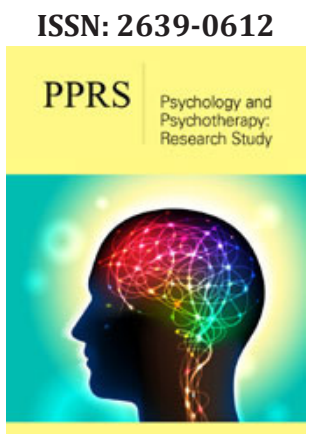

*Corresponding author: Sefa Bulut, Department of Counseling Psychology, Turkey

Submission: 海 August 13, 2020

Published: 温September 11, 2020

Volume 4 - Issue 2

How to cite this article: Sefa Bulut, Beyza Gümüşsoy M. Postpartum Depression and Cognitive Behavioral Therapy from Face to Face Group Sessions to Online Group Sessions. Psychol Psychother Res Stud. 4(2). PPRS. 000581. 2020. DOI: 10.31031/PPRS.2020.04.000581

Copyright@ Sefa Bulut, This article is distributed under the terms of the Creative Commons Attribution 4.0 International License, which permits unrestricted use and redistribution provided that the original author and source are credited.

\author{
Sefa Bulut** and Beyza Güimüşsoy M
}

Department of Counseling Psychology, Turkey

\begin{abstract}
Cognitive behavioral therapy (CBT) is one of the most common used therapy techniques especially among people suffering depression. It was developed by Aeron Beck in 1960s and it has continued to improve itself on different relations with other therapeutic approaches. In addition, cognitive behavioral therapy (CBT) has been used for individual and group therapy sessions. Postpartum depression is a kind of depression which has specific interval of emergence. Almost every stage of pregnancy and after birth is inevitably important to intervene postpartum depression. With the development of technology, online platforms have become more prevalent and common including treatment of postpartum depression as a supplement to face to face therapy sessions. It is important to note that postpartum depression is vital not only mothers experience, but also children's development. This study includes literature review about cognitive behavioral therapy (CBT) and postpartum depression from face to face group sessions to online group session. In the study, it is focused on firstly postpartum depression, cognitive behavioral (CBT) and online cognitive behavioral therapy (CBT) group interventions and finally alternative strategies to cognitive behavioral therapy (CBT) in treatment postpartum depression with different cultural perspectives as well. Outstanding alternatives to cognitive behavioral therapy (CBT) are interpersonal therapy group sessions, peer support groups and music and yoga.
\end{abstract}

Keywords: Postpartum depression; Cognitive behavioral therapy; Face to face group therapy; Online group therapy

\section{Introduction}

To begin with the cognitive behavioral therapy's underlying theory, it is about all psychological disturbances depending on dysfunctional thinking. If people can learn how to evaluate their thinking and change them into more realistic and adaptive way, they can improve their emotional state and behaviors [1]. It means that if people can change their dysfunctional thinking system into more adaptive and constructive format, they would have psychological health. Cognitive behavioral therapy was developed by Aaeron Beck by working on depressed patients. According to his theory, depressed patients have been experienced automatic negative thoughts [1]. CBT for depression focuses on patients identifying, evaluating and responding their depressed thinking, also including negative automatic thought about themselves, worlds and future [1]. Thus, depression started to work with CBT very effectively. Depression can be evaluated in terms of the time period it appears and time interval which is proceed. For instance, postpartum depression has the interval between childbirth and until six months or a year. To develop intervention program to prevent this depression is vital because maternal depression has affected both motherhood experience and baby's relationship with world through mother or caregiver since time of birth. It is important to investigate how it produces, what are the reasons and outcomes for both perspective for researchers who study mother and child relationships. To increase mother and child health, pregnant women should be participated and benefited from treatments for depression specifically on cognitive therapies. This literature review has focused on postpartum depression and different names of it depending on time interval from face to face and online group sessions in terms of CBT 
by including cultural backgrounds as well. In addition to these, it also includes other alternative ways instead of CBT to deal with postpartum depression.

\section{Postpartum Depression}

To give general perspective about depression, in the light of DSM-5, depression criterions are feeling depressed almost all day, decreased in pleasure and interest towards almost many events, changes in weight (loss or sometimes gain), psychomotor agitation or retardation which means moving slowly, feeling fatigue or exhausting in almost all day, decreasing in the level of focusing, thinking about death as well as, feeling hopeless, worthless, and guilt. Finally, at least four criteria must be seen at least two weeks without any history of mania or hypomania to diagnose patient as major depression disorder [2]. Postpartum depression can be explained as an episode of major or minor (sometimes) depressive disorder that emerges during postpartum period. The high risk is from six months after delivery of the child. Postpartum depression risk factors are similar with depression, for extra, including sensitivity to hormone changes [3]. Its symptoms are strong feeling of sadness, anxiety and tiredness that continues for a long time after having baby. Because of these heavy symptoms, women which becomes newly mom cannot take care of themselves and their babies. Postpartum depression generally begins from one to three weeks after giving birth [4]. Postpartum depression has affected several caregiving activities such as feeding practices, safety practices, breastfeeding, sleep routines, the control of vaccinations, and well-child visits [5]. Some authors [6] claims that being successful in breastfeeding and having emotional and practical support from environment including partner and close friends or relatives are significant for mother's well-being and prohibiting depressive symptoms during early postpartum months. Also, they focus on the meaning of being good enough mother which have many different ways to be good enough [6].

Prenatal depression which is general form of postpartum depression. It can be lasted during pregnancy or in the first year of having baby. Prenatal depression, the bounding between mother and fetus during pregnancy, even before birth babies' rhythm and responses cause parents to create baby concept. Especially, baby's personality and sex are very effective to shape this conceptualization. According to a study [7], prenatal bonding between fetus and mother and postnatal depressive symptoms have significantly relationships, which is important to diagnose early postpartum depression [7]. Women are under two-fold risk to develop minor or major depression in prenatal period if they have history of depression. Otherwise, this longitudinal study found that postpartum prevalence figures are lower than literature reports [8]. These studies were related in perspective of mother who have postpartum depression. However, there is also a perspective concerning infants who exposed difficulties and problems in their early life. To better explain, children of mothers experiencing maternal depressive symptoms during from pregnancy to 4 years postpartum are more presumably to have more emotional behavioral difficulties than mothers experiencing lower symptoms
[9]. In addition to postpartum depression (PPD)'s influence on children, maternal depression during postpartum prevents infants' emotional regulation and causes diminished social synchrony, low differentiation of attachment [10]. This is why, postpartum and prenatal depression must be studied and tried to be prevented as soon as possible by taking precautions from states or health insurance. Together with postpartum depression effects on mothers and children, we can obtain information about disorder's prevalence thanks to a study (2015) conducted in US. Just $12 \%$ of pregnant women with depressive disorder in US have accessed mental health care. It suggests that use of mental health care is few among pregnant women, which not makes them a nationally representative sample. The findings of the study are that pregnant women with depression were most presumably being single and more likely to be poor and less educated. Also, they have no consistent insurance coverage [11].

\section{CBT and Online CBT Group Interventions}

According to recent study [12], group based postpartum depression (PPD) interventions are very beneficial to recover mothers. In terms of conclusion of these interventions, environment, sharing and outcomes are three main themes. In respect of qualitative and quantitative research, group-based interventions create a safe environment; thus, mothers with postpartum depression do not feel lonely. Thanks to this group environment, information exchange is provided among members to deal with postpartum depression (PPD) symptoms. Finally, outcomes are seen as mother having higher psychological well-beings and functional capacity [12]. In the light of this recent comprehensive research, we can have a conclusion about group-based intervention being beneficial for mothers with PPD. A metanalysis related with cognitive-behavioral group therapy (CGBT), the level of depression had moderate effect by cognitive-behavioral group therapy. Also, the study claims that depressive patients should complete their course of therapy at least every 6-months [13]. Another study which can be applicable for pregnant or new mother after birth is mindfulness based cognitive behavioral therapy (МBCT). As a result of this study, after 8-week group sessions, level of anxiety and depression get decreased in CBT and MBCT groups [14]. Therefore, depression intervention in general can be applied in postpartum depression with various interactions with CBT.

When it comes to CBT interventions specific to postpartum depression, as a result of systematic review and metanalysis (2012) group cognitive behavioral therapy concluded as more beneficial compared to usual care alone. However, individual CBT experience is more effective than group sessions until to complete 3 months, after 3 months group or individual sessions have same effective level [15]. From a perspective of women experiencing depression after birth, women who have postnatal depression in groups have confronting and ambivalent perceptions and treatments of groups. Afterwards, thanks to group environment, depressed women can gain an understanding about these ambivalent and contrasting perceptions and identify them to change into more adaptive and rational way [16]. In addition to face to face groups sessions, 
internet-based interventions are on the rise as an innovative way. According to a study related with internet-based treatment, they found that patients who can attend treatment responsibly can benefit more from Internet based treatment [17]. Additionally, one of these interventions is therapist-assisted internet cognitive behavior therapy (TAICBT) to deal with postpartum depression. To the extent of participants responses, TAICBT intervention program is afforded flexibility, accessibility, convenience and anonymity as well as privacy thanks to internet-based program [18]. The program provides participants to choose correct direction, raise self-awareness and parenting skills. In addition to this, having internet therapist make their treatment more individualized and supportive. For further investigation, internet based cognitive therapy method can be used to help women with postpartum depression and postnatal depression [18].

One of the internet-based intervention programs is "Be A Mom (2018)". It is an e-health intervention program for postpartum depression includes both psychoeducation and psychotherapy based on cognitive therapies. By this web-based intervention program, participants obtained healthy and necessary literature knowledge about postpartum depression. Also, they acquired some strategies and acceptance of difficulties in future because of postpartum depression. Furthermore, they concentrated on dyadic issues such as couple's satisfaction with each other and communication skills as apart from literature in terms of PPD's factors. Use of web-based program becomes beneficial to prevent PPD [19]. A study [20] focused to help depressed mother who are in economically disadvantaged group claims that women who attending "Mom-Net" intervention program in high level reported low scores in depression. Mom-Net intervention is also based on CBT including online tutorials and weekly couch followings with phone calls [20]. Mothers' responses about program are like having good understanding of materials, the website easy to use and coaches to be supportive [20].

Also, another study was conducted with low-income mothers' in-home CBT visiting and depression. There is not significantly difference in depression reduction among low-income mothers in home visiting and parenting stress, nurturing parenting and child adjustment. However, they found that depression was significantly related with parenting stress and nurturing parents [21]. Mother with depression taking in home-CBT and home visiting at the same time were likely to be superior to only mother with depression taking home visiting alone [22]. Moreover, online interventions are useful to aid women who are under PPD risk. Internet use hinders various barriers such as lack of time to attend groups sessions, unwilling for meditation, shame because of PPD, and the cost of the treatment [23]. However, there are some disadvantages of online therapies. Although online interventions are useful to aid women who are under PPD risk. A study [24] comparing face to face and online cognitive behavioral therapy in terms of depression concluded that working alliance in online and face to face therapy can be compared as almost same. However, in online setting anonymous online therapeutic relationship is not more stable than face to face therapy settings. Therefore, it means that participants in online setting can stop continuing session by just disappearing which makes online setting weaker [24]. Therefore, even if there are some negative aspects of online therapy sessions, we can reach a conclusion that face to face therapy can be high probably transformed into online therapy sessions in terms of cognitive therapies in future. If people become willing to participate and establish strong therapeutic alliances by high attendance, platforms online or face to face does not matter, connections and exchange of information can be provided. By taking into consideration cultural differences among mothers in terms of postnatal depression, a brief-group intervention program based on CBT was used among Chinese pregnant women to decrease the level of depression during the pregnancy [25]. According to results of the study, it was found that CBT is evidence-based intervention program to prevent postnatal depression among Chinese pregnant women [25]. Additionally, another culturally different study was conducted by Iranian mothers with postpartum depression. Study has two interventions as debriefing including discussing mothers' labor, birth, post-delivery experiences and brief cognitive behavioral therapy including methods for managing anxiety, social skills, problem solving, relaxation and cognitive coping. According to result of the study, they found mothers having traumatic childbirth with two interventions have low depression mean scores 3-months post-delivery rather than control groups [26].

As supplement to mother-infant relationship in relation with postpartum depression, However, there is another component of this dyadic relationship "father". Unfortunately, studies commonly have concentrated on mother-child relationship, by skipping father. A study [27] focused on this missing component in literature. According to their statements, in order to obtain perfect medical care, family units must be taken into consideration including father too, not just focusing on mother-infant dyads. Since father with depression after the birth of their child was related with the personal history of depression and interaction with partners' depression during pregnancy [27].

\section{Alternative Strategies to CBT in Treatment PPD}

First of all, we can categorize alternatives into three subcategories as support groups, music and yoga, as well as interpersonal therapy. Women with postpartum depression engaging support groups are experienced being supported and shared their symptoms with other women experiencing same problem with them without feeling judgmental via support groups [28]. At. the same time, these groups members made mediation instead of antidepressants. Furthermore, women in support groups with PPD accepted their sickness by telling their own stories, got stronger by confirmation of caring experiences. Also, they experienced new practice ways and are encouraged by support group by observing their improvement [29]. Although this study is not representative, its contribution to literature is inevitable in terms of women's need for self-determination in the PPD process, and genuine support with their companions [29]. As final, In the perspective of short-term group experiences, a study [30] claims that women's scores on Edinburgh Postnatal Depression Scale 
attending short term group psychotherapy were diminished for the risk [30].

Secondly, from interesting perspective, researcher [31] studied on music and intersubjectivity of mother-infant in the context of postpartum depression. The result is that mother with postpartum depression-infant provide development from animated atmosphere to create them being lively, vital and joyful [31]. This study contributes a different combination with postpartum depression patients. As well as, for diverse and cost-effective group experience to deal with postpartum depression, yoga and support groups decreased depression, anxiety, anger, development in quality of relationship and cortisol levels [32]. Thirdly, another alternative way of CBT is interpersonal group therapy which works focused and brief to dealt with PPD. Provision of peer support, validation and encouragement are main factors of therapy. Also, social isolation decreased by experiencing catharsis and changing themselves as a taking role in cohesive group [33]. According to finding of groups sessions CBT with interaction interpersonal therapy, all group members with prenatal depression got rid of suicidal thoughts after completed groups and members with dealing with problems in continuing their career had a chance to return their work after groups sessions [34]. Also, mother participating interpersonal group therapy have enhancing in intimate relationship with their partners. Moreover, they have better bond with their infants thanks to interpersonally intervention [35].

\section{Conclusion}

To sum up, cognitive behavioral therapy (CBT) intervention program is beneficial, time-limited and structured to treat patient with postpartum depression. Especially, with the developments in technology, online platforms have become more prevalent and common. These developments' effects reflect on therapeutic relationship with patients. Online individual therapy and group therapy could be preferable among mothers who have immediate responsibilities to their children like breastfeeding. In addition to being easy-accessible, online therapies could be chosen by mothers who have low economic status because the cost of the online therapy become more appropriate. Beside positive outcomes, there are also negative outcomes for online CBT therapy instead of face to face therapy. For instance, people can be just disappeared by stopping participating. However, if the participants take it seriously, it does not make any difference between online or face to face therapy in terms of cognitive behavioral therapy. Working alliance and group cohesiveness can improve as much as face to face therapy. This is good for every woman suffering depression after childbirth. Actually, COVID-19 pandemic process is able to make improvements to pass online therapies and to make them more common. Researches and studies related online therapy recommend for future studies as well. Furthermore, there various alternative ways having evidence based to treat postpartum depression such as using intersubjectivity with music between mother-infant, practice yoga and meditation. Finally, interpersonal therapy as another theory which is beneficial to deal with depression.

\section{References}

1. Beck JS (2011) CBT: Basics and beyond. The Guilford Press.

2. American Psychiatric Association (2013) Diagnostic and statistical manual of mental disorders (DSM-5®). American Psychiatric Pub.

3. Ohara MW, McCabe JE (2013) Postpartum depression: Current status and future directions. Annu Rev Clin Psychol 9: 379-407.

4. March of Dimes (2019) Postpartum depression.

5. Field T (2010) Postpartum depression effects on early interactions, parenting, and safety practices: A review. Infant Behavior and Development 33(1): 1-6.

6. Haga SM, Lynne A, Slinning K, Kraft P (2012) A qualitative study of depressive symptoms and well-being among first-time mothers. Scand J Caring Sci 26(3): 458-466.

7. Dubber S, Reck C, Müller M, Gawlik S (2015) Postpartum bonding: the role of perinatal depression, anxiety and maternal-fetal bonding during pregnancy. Arch Womens Ment Health 18(2): 187-195.

8. Banti S, Mauri M, Oppo A, Borri C, Rambelli C, et al. (2011) From the third month of pregnancy to 1 year postpartum. Prevalence, incidence, recurrence, and new onset of depression. Results from the Perinatal depression-research \& screening unit study. Compr Psychiatry 52(4): 343-351.

9. Giallo R, Woolhouse H, Gartland D, Hiscock H, Brown S (2015) The emotional-behavioural functioning of children exposed to maternal depressive symptoms across pregnancy and early childhood: A prospective Australian pregnancy cohort study. Eur Child Adolesc Psychiatry 24(10): 1233-1244.

10. Granat A, Gadassi R, Schechtman GE, Feldman R (2017) Maternal depression and anxiety, social synchrony, and infant regulation of negative and positive emotions. Emotion 17(1): 11-27.

11. Byatt N, Xiao RS, Dinh KH, Waring ME (2016) Mental health care use in relation to depressive symptoms among pregnant women in the USA. Archives of Women's Mental Health 19(1): 187-191.

12. Gillis BD, Parish AL (2019) Group-based interventions for postpartum depression: An integrative review and conceptual model. Arch Psychiatr Nurs 33(3): 290-298.

13. Feng CY, Chu H, Chen CH, Chang YS, Chen TH, et al. (2012) The effect of cognitive behavioral group therapy for depression: A meta-analysis 2000-2010. Worldviews on Evidence-Based Nursing 9(1): 2-17.

14. Manicavasgar V, Parker G, Perich T (2011) Mindfulness-based cognitive therapy vs cognitive behaviour therapy as a treatment for nonmelancholic depression. J Affect Disord 130(1-2): 138-144.

15. Huntley AL, Araya R, Salisbury C (2012) Group psychological therapies for depression in the community: Systematic review and metaanalysis. Br J Psychiatry 200(3): 184-190.

16. Scope A, Booth A, Sutcliffe P (2012) Women's perceptions and experiences of group cognitive behaviour therapy and other group interventions for postnatal depression: A qualitative synthesis. J Adv Nurs 68(9): 1909-1919.

17. Bendelin N, Hesser H, Dahl J, Carlbring P, Nelson KZ, et al. (2011) Experiences of guided internet-based cognitive-behavioural treatment for depression: a qualitative study. BMC Psychiatry 11(1): 107.

18. Pugh NE, Hadjistavropoulos HD, Hampton AJ, Bowen A, Williams J (2015) Client experiences of guided internet cognitive behavior therapy for postpartum depression: A qualitative study. Arch Womens Ment Health 18(2): 209-219.

19. Fonseca A, Pereira M, Araújo Pedrosa A, Gorayeb R, Ramos MM, et al. (2018) Be a mom: Formative evaluation of a web-based psychological 
intervention to prevent postpartum depression. Cognitive and Behavioral Practice 25(4): 473-495.

20. Sheeber LB, Seeley JR, Feil EG, Davis B, Sorensen E, et al. (2012) Development and pilot evaluation of an Internet-facilitated cognitivebehavioral intervention for maternal depression. J Consult Clin Psychol 80(5): 739.

21. Ammerman RT, Altaye M, Putnam FW, Teeters AR, Zou Y, et al. (2015) Depression improvement and parenting in low-income mothers in home visiting. Arch Womens Ment Health 18(3): 555-563.

22. Ammerman RT, Putnam FW, Altaye M, Stevens J, Teeters AR, et al. (2013) A clinical trial of in-home CBT for depressed mothers in home visitation. Behavior Therapy 44(3): 359-372.

23. Maloni JA, Przeworski A, Damato EG (2013) Web recruitment and internet use and preferences reported by women with postpartum depression after pregnancy complications. Arch Psychiatr Nurs 27(2): 90-95.

24. Preschl B, Maercker A, Wagner B (2011) The working alliance in a randomized controlled trial comparing online with face-to-face cognitive-behavioral therapy for depression. BMC Psychiatry 11(1): 189.

25. Leung SS, Lee AM, Chiang VC, Lam SK, Kuen YW, et al. (2013) Culturally sensitive, preventive antenatal group cognitive-behavioural therapy for Chinese women with depression. Int J Nurs Pract 1: 28-37.

26. Abdollahpour S, Keramat A, Mousavi SA, Khosravi A (2018) The effect of debriefing and brief cognitive-behavioral therapy on postpartum depression in traumatic childbirth: A randomized clinical trial. Journal of Midwifery and Reproductive Health 6(1): 1122-1131.
27. Edward KL, Castle D, Mills C, Davis L, Casey J (2015) An integrative review of paternal depression. Am J Mens Health 9(1): 26-34.

28. Cook C, Goyal D, Allen M (2019) Experiences of women with postpartum depression participating in a support group led by mental health providers. MCN Am J Matern Child Nurs 44(4): 228-233.

29. Montgomery P, Mossey S, Adams S, Bailey PH (2012) Stories of women involved in a postpartum depression peer support group. Int J Ment Health Nurs 21(6): 524-532.

30. Pessagno RA, Hunker D (2013) Using short-term group psychotherapy as an evidence-based intervention for first-time mothers at risk for postpartum depression. Perspect Psychiatr Care 49(3): 202-209.

31. Van Puyvelde M, Rodrigues H, Loots G, De Coster L, Du Ville K, et al. (2014) Shall we dance? Music as a port of entrance to maternal-infant intersubjectivity in a context of postnatal depression. Infant Ment Health J 35(3): 220-232.

32. Field T, Diego M, Delgado J, Medina L (2013) Yoga and social support reduce prenatal depression, anxiety and cortisol. Journal of Bodywork and Movement Therapies 17(4): 397-403.

33. Reay RE, Mulcahy R, Wilkinson RB, Owen C, Shadbolt B, et al. (2012) The development and content of an interpersonal psychotherapy group for postnatal depression. Int J Group Psychother 62(2): 221-251.

34. Goldvarg E, Kissen M (2011) Group psychotherapy for women suffering from postpartum depression. Group pp. 235-246.

35. Mulcahy R, Reay RE, Wilkinson RB, Owen C (2010) A randomized control trial for the effectiveness of group interpersonal psychotherapy for postnatal depression. Arch Womens Ment Health 13(2): 125-139. 